\title{
A low cortisol response to stress is associated with musculoskeletal pain combined with increased pain sensitivity in young adults: a longitudinal cohort study
}

Markus Paananen ${ }^{1 *}$, Peter O'Sullivan ${ }^{2}$, Leon Straker ${ }^{2}$, Darren Beales ${ }^{2}$, Pieter Coenen ${ }^{2}$, Jaro Karppinen ${ }^{1,3}$, Craig Pennell ${ }^{4}$ and Anne Smith ${ }^{2}$

\begin{abstract}
Background: In this study, we investigated whether an abnormal hypothalamic-pituitary-adrenal (HPA) axis response to psychosocial stress at 18 years of age is associated with musculoskeletal (MS) pain alone and MS pain combined with increased pain sensitivity at 22 years of age.

Methods: The study sample included 805 participants from the Western Australian Pregnancy Cohort (Raine) Study who participated in the Trier Social Stress Test (TSST) at age 18 years. Number of pain sites, pain duration, pain intensity and pain frequency were assessed at age 22 to measure severity of MS pain. Cold and pressure pain thresholds were determined at age 22. Group-based trajectory modeling was applied to establish cortisol response patterns based on the TSST. Logistic regression was used to study the association of TSST patterns with MS pain alone and MS pain combined with increased cold or pressure pain sensitivity, adjusted for relevant confounding factors. All analyses were stratified by sex.
\end{abstract}

Results: The mean (standard deviation) age during the TSST was 18.3 (0.3) years, and during MS pain assessment it was 22.2 (0.6). Forty-five percent of the participants were female. Three cortisol response patterns were identified, with cluster 1 (34\% of females, $21 \%$ of males) reflecting hyporesponse, cluster 2 (47\%, $54 \%$ ) reflecting intermediate response and cluster $3(18 \%, 24 \%)$ reflecting hyperresponse of the HPA axis. MS pain was reported by $42 \%$ of females and $33 \%$ of males at age 22 years. Compared with females in cluster 2, females in cluster 1 had an increased likelihood of having any MS pain (odds ratio 2.3, $95 \%$ confidence interval 1.0-5.0) and more severe MS pain $(2.8,1.1-6.8)$ if their cold pain threshold was above the median. In addition, females in cluster 1 had an increased likelihood $(3.5,1.3-9.7)$ of having more severe MS pain if their pressure pain threshold was below the median. No statistically significant associations were observed in males.

Conclusions: This study suggests that a hyporesponsive HPA axis at age 18 years is associated with MS pain at 22 years in young females with increased pain sensitivity.

Keywords: Musculoskeletal, Hypersensitivity, Quantitative sensory testing, Cortisol, Trajectory modeling

\footnotetext{
* Correspondence: markus.paananen@oulu.fi

${ }^{1}$ Centre for Life Course Epidemiology, and Medical Research Centre Oulu,

Oulu University Hospital and University of Oulu, Oulu, Finland

Full list of author information is available at the end of the article
}

( Biomed Central (c) 2015 Paananen et al. Open Access This article is distributed under the terms of the Creative Commons Attribution 4.0 International License (http://creativecommons.org/licenses/by/4.0/), which permits unrestricted use, distribution, and reproduction in any medium, provided you give appropriate credit to the original author(s) and the source, provide a link to the Creative Commons license, and indicate if changes were made. The Creative Commons Public Domain Dedication waiver (http://creativecommons.org/publicdomain/zero/1.0/) applies to the data made available in this article, unless otherwise stated. 


\section{Background}

Chronic musculoskeletal (MS) pain is a disabling condition that occurs without an apparent tissue-level pathology in several functional pain disorders [1]. Its prevalence is higher among females and psychologically stressed individuals [2]. Recent findings suggest a mediating role of a major stress-regulating system, the hypothalamic-pituitary-adrenal (HPA) axis, in the association of stress with pain and pain hypersensitivity [3-5]. Several associations between variations in genes related to both HPA axis and widespread MS pain have been observed, emphasizing their potential link [6].

The HPA axis is a major system involved in the maintenance of homeostasis during stress, but it may develop first hyper- and then hypoactivity in response to chronic stress [7]. There is growing evidence that relative hypocortisolism, as a marker of stress-induced HPA axis dysfunction, may in turn increase vulnerability to pain and chronic pain disorders [5, 8-13]. In an experimental study, pharmacologically induced hypocortisolism increased mechanical pain sensitivity and potentiated hyperalgesia in healthy males, suggesting that HPA axis alterations have a causal role in pain [5]. In a crosssectional study of female twins, a blunted cortisol diurnal pattern was associated with higher perceived pain intensity during a cold pressor test [13].

A cross-sectional population-based study found that chronic multi-site MS pain was associated with lower cortisol levels and a blunted diurnal cortisol pattern [8]. However, the same authors did not find an association between HPA axis functioning and the onset of chronic multi-site MS pain 6 years later [14]. Researchers in a longitudinal study found that a flattened diurnal cortisol profile and high post-dexamethasone cortisol levels predicted the onset of chronic multi-site MS pain 15 months later among individuals with high somatization and health-seeking behaviour [10].

Relative hypocortisolism may be present already in early childhood in association with early-life stress and psychopathology [15]. Similarly, chronic MS pain is reported already in adolescence [16], implying that the disorder may also begin early in life in some individuals. However, the association between HPA axis function and MS pain is still unclear among adolescents and young adults who are generally healthy and highly sensitive to stress [17].

Therefore, the aim of this population-based study was to investigate whether HPA axis response during acute psychosocial stress at 18 years of age was associated with subsequent MS pain presence and severity at age 22 years. In particular, we were interested in analysing the association between cortisol responses with high levels of MS pain in addition to high pain sensitivity. As such, we performed two set of analyses to evaluate (1)
MS pain alone and (2) MS pain combined with increased cold pain threshold (CPT) and decreased pressure pain threshold (PPT). On the basis of previous research, we hypothesized that (1) hyporeactive HPA axis at 18 years would be associated with more severe MS pain at 22 years and (2) the association would be stronger in the presence of relative cold and pressure pain hypersensitivity.

\section{Methods}

\section{Study sample and data collection}

The study sample was from the Western Australian Pregnancy Cohort (Raine) Study, which includes children of pregnant women enrolled in the study at or before the 18th gestation week between 1989 and 1992 . The original purpose of the study was to examine the effects of frequent and repeated ultrasound scans on pregnancy outcomes. The study is described in more detail elsewhere [18]. The population-based cohort originally included 2868 children who have been comprehensively followed from birth to 22 years of age with multiple follow-up points. The inclusion criteria of the study were gestational age between 16 and 20 weeks, sufficient English proficiency and an intention to remain in Western Australia. The cohort has been shown to be a socioeconomically representative sample of the Western Australian population [19]. The Raine Study ethical approval for the study was obtained from the University of Western Australia Human Research Ethics Committee (HREC), the Curtin University HREC for the 22-year-old cohort follow-up and the Princess Margaret Hospital for Children HREC for the 17-year-old cohort follow-up. Written informed consent was obtained from all participants.

At 17 years of age, 1399 adolescents provided questionnaire data including physical activity level and depressive symptoms, and measured height and weight were also collected. At 18 years of age, 1137 individuals participated in the Trier Social Stress Test (TSST); completed a questionnaire concerning illnesses, medication use, smoking and oral contraceptive (OC) use; and had their height and weight measured. Data on MS pain and pain sensitivity were collected at 22 years of age, when 1138 cohort members answered the Örebro Musculoskeletal Pain Questionnaire and participated in clinical assessments, including quantitative sensory testing. All active cohort members were invited to participate in each follow-up. The reason for nonparticipation at each time point was primarily unwillingness to participate.

In total, 216 participants declined to have blood collected during the TSST and 116 were excluded because of inability to complete the TSST $(n=2)$, unusable cortisol samples $(n=3)$, severe menstrual pain $(n=1)$, pregnancy $(n=2)$, lactation $(n=2)$, type 1 diabetes $(n=4)$, use of exogenous steroids $(n=7)$, neuroactive 
medications $(n=22)$, antidepressants $(n=19)$ or other medications affecting the HPA axis $(n=2)$, noncompletion of the full test $(n=15)$ or having younger siblings participating in the study $(n=37)$. After exclusions, 366 females and 439 males had complete data on plasma total cortisol, and 277 females and 280 males had data on both MS pain and cortisol. The number of individuals included in the analysis varied from 198 to 222 among females (55 missing for covariates, 22 missing for cold pain threshold and 24 missing for pressure pain threshold) and from 198 to 215 among males (65 missing for covariates, 17 missing for cold pain threshold and 11 missing for pressure pain threshold), as only individuals with complete data on the measures of interest were included in the analysis. Because this study involved three waves of follow-up (at ages 17, 18 and 22 years), most of the missing data is due to participants choosing not to participate in one or more of these follow-ups. The other cause was incomplete data on the various questionnaires or tests.

\section{Trier Social Stress Test}

HPA axis function was evaluated by the TSST, which includes items assessing the essential features of a reliable stressor being both uncontrollable and social-evaluative $[20,21]$. It is a highly standardized protocol to induce psychological stress and cortisol response [20, 22]. The subjects arrived at the hospital between $1200 \mathrm{~h}$ and $1600 \mathrm{~h}$ for the TSST. They were informed that eating and/or drinking anything other than water within $1 \mathrm{~h}$ before the test would affect stress hormone levels and that therefore the test was to be conducted at least $1 \mathrm{~h}$ after eating. Within 15 min after arrival, an anaesthetist inserted an intravenous cannula after permission for taking blood samples was obtained. During the next 45-min rest period, the participants completed a questionnaire, and their height and weight were measured. After the rest period, the participants performed a 15-min stress test that included a free speech interview and an arithmetic task in front of a non-responsive panel of two interviewers and a dummy camera. In the debriefing discussion afterwards, the goal of the study and the nature of the stressor were explained to the participants. In total, eight blood samples were taken: just preceding the test ( $0 \mathrm{~min})$; after completing the test (15 minutes); and at $25,35,45,60,75$ and $105 \mathrm{~min}$.

Blood samples were collected in ethylenediaminetetraacetic acid tubes (BD Biosciences, San Jose, CA, USA) and stored on ice during the test and were immediately centrifuged, aliquoted and frozen at $-80{ }^{\circ} \mathrm{C}$ after the test until analyses. The GammaCoat ${ }^{\mathrm{m}}$ Plasma Renin Activity ${ }^{125}$ I cortisol radioimmunoassay kit (DiaSorin, Stillwater, MN, USA) was used for quantifying total plasma cortisol, and concentrations in micrograms per decilitre were converted to nanomoles per litre by multiplying by 27.59. All assays were performed in duplicate against a standard curve and repeated where required. The intra- and inter-assay variability was acceptable $(<10 \%)$ for all assays.

\section{Musculoskeletal pain}

Pain status was evaluated using five items concerning pain experience from the Örebro Musculoskeletal Pain Questionnaire [23]; we did not use the full questionnaire, as it measures likelihood of developing a persistent pain problem and related disability. The participants reported whether they had current pain in the neck, left and/or right shoulder, left and/or right arm, upper and/ or lower back, left and/or right leg or other site. They were asked about the duration of their pain with ten answer options varying from ' 0 days' to 'over 1 year' that were scored on a corresponding 0-9 scale. They were asked about their pain intensity during the past week, average pain intensity in the past 3 months and average frequency of pain episodes during the past 3 months with ten numerical answer options from 'no pain' or 'never' to 'pain as bad as it could be' or 'always' scored on a corresponding 0-9 scale. A pain problem severity index describing the severity of pain problems and varying from 0 to 37 was calculated by adding the following scores: a sum of pain sites (0-10), pain duration (0-9), mean of two pain intensity measures (0-9) and pain frequency (0-9). Two dichotomous variables were derived by categorizing subjects as follows: (1) no pain at all versus pain at any site and (2) pain problem severity index above and below the 75 th percentile.

\section{Quantitative sensory testing}

CPT and PPT were measured in a clinical setting according to a standardized protocol [24]. CPT was assessed using a Peltier element-based thermode with a $12.5-\mathrm{cm}^{2}$ probe (Modular Sensory Analyser; Somedic AB, Hörby, Sweden) applied on the skin of the dorsal wrist. The baseline temperature was $32{ }^{\circ} \mathrm{C}$, and the cutoff minimum temperature limit was $5{ }^{\circ} \mathrm{C}$. The rate of temperature change was set to $1{ }^{\circ} \mathrm{C} /$ second, and the stimulus was terminated when the subject pressed a button. The participants were given the following instructions: Allow the temperature to drop until the moment it reaches a point where it feels uncomfortably or painfully cold, and then press the button. This means the very first onset of discomfort or pain and not the most cold that you can bear'. To measure PPT, an algometer (Somedic AB, Farsta, Sweden) with a $1-\mathrm{cm}^{2}$ contact head was applied perpendicularly to the skin at four standardized test sites in the following sequence: (1) dorsal wrist (middle of the dorsal aspect of the wrist joint line), (2) leg (the muscle belly of tibialis anterior, approximately $2.5 \mathrm{~cm}$ lateral and $5 \mathrm{~cm}$ distal to the tibial tubercle), (3) 
cervical spine (the trapezius muscle, at the mid-point between the $\mathrm{C} 7$ spinous process and the lateral acromion) and (4) lumbar spine (at the erector spinae, $2 \mathrm{~cm}$ lateral to the L4-L5 interspinous space). The pressure was increased with a rate of $50 \mathrm{kPa} /$ second, and the cut-off maximum limit was $1000 \mathrm{kPa}$. The following instructions were given: 'The moment the pressure increases to a point where it first feels uncomfortable or painful, press and release the button. This means the very first onset of discomfort or pain and not the most pressure that you can bear'. CPT and each PPT were tested four times, and the mean threshold value of the last three measurements was calculated (at most one missing value allowed). The sex-specific median values were used as cut-off points to identify individuals with increased pain sensitivity [25]. CPT value above the median indicated increased cold pain sensitivity, and an average of PPT values at four different sites below the median indicated increased pressure pain sensitivity.

\section{Covariates}

OC use, smoking, body mass index (BMI), physical activity level and depressive symptoms were measured at 17 or 18 years of age and selected as covariates, as each of them has been related to HPA axis function and MS pain in previous studies [26-30]. OC use (yes or no) and smoking (yes or no) were inquired about during the TSST session and dichotomized into non-users vs. users and current smokers vs. non-smokers. BMI was measured and calculated as weight (in kilograms) divided by the square of body length (square meters) and categorized into normal $(\mathrm{BMI}<25)$, overweight $(25 \geq \mathrm{BMI}<30)$ and obese (BMI $\geq 30$ ). If $\mathrm{BMI}$ at age 18 was missing, height and weight data from 17 years of age were used and BMI was categorized using the International Obesity Task Force age-specific cut-off points [31]. Participation in moderate to vigorous physical activity was measured using the short-form International Physical Activity Questionnaire [32]. As the association of physical activity with presence and severity of MS pain was U-shaped, the study subjects were categorized into three groups: (1) $0 \mathrm{~h} /$ week, (2) $0.1-4.9 \mathrm{~h} /$ week and (3) $5.0 \mathrm{~h} /$ week or more. The Beck Depression Inventory was used to assess depressive symptoms [33], and individuals were classified using the recommended cut-off values: minimal, 0-13; mild, 14-19; and moderate or severe, 20-63.

\section{Statistical analyses}

The cortisol data were analysed using the group-based trajectory modeling (GBTM) approach, which is an application of the finite mixture models for longitudinal data [34]. The aim of GBTM is to identify the smallest number of latent groups following different response patterns, to which individuals are assigned probabilistically.
The selection of analytical method was based on the assumption that there are distinct patterns of change in cortisol levels over time. Specifically, in light of previous findings and the allostatic load theory $[4,6]$, we anticipated identifying groups with atypical cortisol response (i.e., with hypo- or hyper-reactive HPA axis). Our purpose was to summarize the complex cortisol data in a parsimonious manner, as done in previous studies with GBTM using time-based cortisol data [35, 36].

As there are sex differences in pain and cortisol responses, males and females were modelled separately $[17,26,29]$. The GBTM with one to five latent groups was assessed in both sexes. The censored normal distribution was selected for the modelling after transforming the cortisol values logarithmically. To determine the number of groups that best represented the data heterogeneity, the Bayesian information criterion (BIC) was used; we also considered the practical value of the model with respect to the research question, as recommended by Nagin and Odgers [37]. The $x^{2}$ test was used to compare the covariates used in the multivariate models across the cortisol response groups.

Logistic regression analysis was applied to model the association between MS pain and cortisol response groups, with adjustments made for all potentially confounding variables and stratification by sex. Only the individuals with full data on MS pain, cortisol and covariates were included in the models. The association was first analysed using (1) pain at any site or (2) pain problem severity index above the 75th percentile as an outcome variable, with the reference group including those without or low severity of pain. In the next phase, four composite outcomes were created in light of (1) previous research in pain sensitivity testing, which does not provide clear recommended cut-off values for CPT or PPT for this age group; and (2) the need to identify those participants with the most severe cases with sufficient sample sizes in each subgroup; and (3) the need to combine pain sensitivity and pain problem severity as an outcome. The outcomes were (1) pain at any site and CPT above the median, (2) pain at any site and PPT below the median, (3) pain problem severity index above the 75th percentile and CPT above the median and (4) pain problem severity index above the 75th percentile and PPT below the median. In the analyses regarding composite variables, the reference group comprised those without pain or with low severity of pain as well as those with pain but with pain sensitivity below the median. Previously, composite outcomes have been used in the studies of, for example, cardiovascular, pulmonary and gastrointestinal diseases [38], as well as in pain research [39]. All analyses were performed using STATA 13.1 software (StataCorp, College Station, TX, USA). 


\section{Results}

The sample included 366 females and 439 males with a mean (standard deviation [SD]) age of $18.3(0.3)$ years during the TSST and a mean age of 22.2 (0.6) during MS pain assessment. The distributions of variables used in the study are shown in the Table 1.

\section{Musculoskeletal pain}

MS pain at any site was reported by $42 \%$ of females and $33 \%$ of males (Table 1 ). The pain problem severity indexes ranged from 0 to 33 among females and from 0 to 29 among males, and the mean scores were 17 (SD 7) among females and 13 (SD 6) among males having any MS pain. Females with a score of 15 or more belonged to the highest quartile, whereas for males the 75th percentile was 9, hereafter referred to as high pain problem severity index. CPT was above the median (females $15{ }^{\circ} \mathrm{C}$ or more, males $7{ }^{\circ} \mathrm{C}$ or more) in $54 \%$ of females and $48 \%$ of males with any pain, and in $59 \%$ of females and $48 \%$ of males with a high pain problem severity index. PPT below the median (females $318 \mathrm{kPa}$ or less, males $419 \mathrm{kPa}$ or less) was found in $54 \%$ of females and $45 \%$ of males with any pain and in $49 \%$ of females and $47 \%$ of males with a high pain problem severity index.

\section{Cortisol response groups}

The three-group model was considered to fit the data best in both sexes. It showed substantially lower BIC values (i.e., a better fit) than the models with one or two groups and the sizes of groups were reasonable providing meaningful interpretation of the data. Each individual had a high probability of belonging to their allocated group, confirming model adequacy, with average posterior probabilities being 0.97 for cluster 1, 0.97 for cluster 2, and 0.97 for cluster 3 among females, and 0.96, 0.97, and 0.96 among males, respectively.

The mean baseline, peak and minimum cortisol values over the study period were the highest in cluster $3(764 / 947 / 584 \mathrm{mmol} / \mathrm{L}$, respectively, in females; $577 / 760 / 373 \mathrm{mmol} / \mathrm{L}$, respectively, in males), followed by cluster $2(392 / 523 / 280 \mathrm{mmol} / \mathrm{L}$, respectively; 344/ $485 / 233 \mathrm{mmol} / \mathrm{L}$, respectively) and cluster 1 (227/ 295/152 mmol/L, respectively; 225/301/150 mmol/L, respectively). Cluster 1 , including $34 \%$ of females and $21 \%$ of males, had only a slight change in cortisol, with small differences between baseline and peak (difference between baseline and peak $68 \mathrm{mmol} / \mathrm{L}$ in females, $76 \mathrm{mmol} / \mathrm{L}$ in males) (Figs. 1 and 2). Cluster 2 , including $47 \%$ of females and $54 \%$ of males, showed an immediate cortisol response followed by a consistent return to baseline, and peak values differed clearly from baseline $(128 \mathrm{mmol} / \mathrm{L}, 140 \mathrm{mmol} / \mathrm{L})$. Cluster 3, including $18 \%$ of females and $24 \%$ of
Table 1 Distribution of variables used in the study

\begin{tabular}{|c|c|c|}
\hline & Females, $n(\%)$ & Males, $n(\%)$ \\
\hline \multicolumn{3}{|l|}{ Cortisol response group } \\
\hline Cluster 1 & $126(34)$ & $94(21)$ \\
\hline Cluster 2 & $173(47)$ & $238(54)$ \\
\hline Cluster 3 & $67(18)$ & $107(24)$ \\
\hline \multicolumn{3}{|l|}{ Oral contraceptive use } \\
\hline No & $227(62)$ & \\
\hline Yes & $139(38)$ & \\
\hline \multicolumn{3}{|l|}{ Smoking } \\
\hline No & $324(89)$ & $355(82)$ \\
\hline Yes & $41(11)$ & $79(18)$ \\
\hline \multicolumn{3}{|l|}{ Body mass index } \\
\hline Normal & $261(72)$ & $313(72)$ \\
\hline Overweight & $58(16)$ & $79(18)$ \\
\hline Obese & $43(12)$ & $41(10)$ \\
\hline \multicolumn{3}{|l|}{ Physical activity level } \\
\hline $0 \mathrm{~h} / \mathrm{wk}$ & $139(45)$ & $108(32)$ \\
\hline $0.1-4.9 \mathrm{~h} / \mathrm{wk}$ & $112(37)$ & $114(34)$ \\
\hline$\geq 5.0 \mathrm{~h} / \mathrm{wk}$ & $56(18)$ & $117(35)$ \\
\hline \multicolumn{3}{|l|}{ Depressive symptoms } \\
\hline Minimal & $219(72)$ & $309(86)$ \\
\hline Mild & $42(14)$ & $33(9)$ \\
\hline Moderate to severe & $42(14)$ & $16(5)$ \\
\hline \multicolumn{3}{|l|}{ MS pain } \\
\hline No & $160(58)$ & $188(67)$ \\
\hline Yes & $117(42)$ & $92(33)$ \\
\hline \multicolumn{3}{|l|}{ MS pain and CPT $>P 50^{\mathrm{a}}$} \\
\hline No & $193(77)$ & $218(85)$ \\
\hline Yes & $57(23)$ & $37(15)$ \\
\hline \multicolumn{3}{|l|}{ MS pain and $\mathrm{PPT}<\mathrm{P} 50^{\mathrm{b}}$} \\
\hline No & $189(76)$ & $227(86)$ \\
\hline Yes & $59(24)$ & $37(14)$ \\
\hline \multicolumn{3}{|c|}{ Pain problem severity index ${ }^{c}$} \\
\hline Low & $204(74)$ & $210(75)$ \\
\hline $\mathrm{High}^{c}$ & $73(26)$ & $70(25)$ \\
\hline \multicolumn{3}{|c|}{ High pain ${ }^{c}$ and $C P T>P 50^{a}$} \\
\hline No & $215(86)$ & $228(89)$ \\
\hline Yes & $35(14)$ & $27(11)$ \\
\hline \multicolumn{3}{|c|}{ High pain ${ }^{c}$ and $\mathrm{PPT}<\mathrm{P} 50^{\mathrm{b}}$} \\
\hline No & $219(88)$ & $235(89)$ \\
\hline Yes & $29(12)$ & $29(11)$ \\
\hline
\end{tabular}

MS musculoskeletal, CPT cold pain threshold, $P P T$ pressure pain threshold

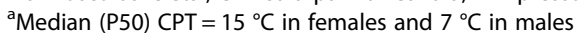
${ }^{b}$ Median (P50) PPT $=318 \mathrm{kPa}$ in females and $419 \mathrm{kPa}$ in males ${ }^{C}$ High pain problem severity index $=15$ or more in females and 9 or more in males 


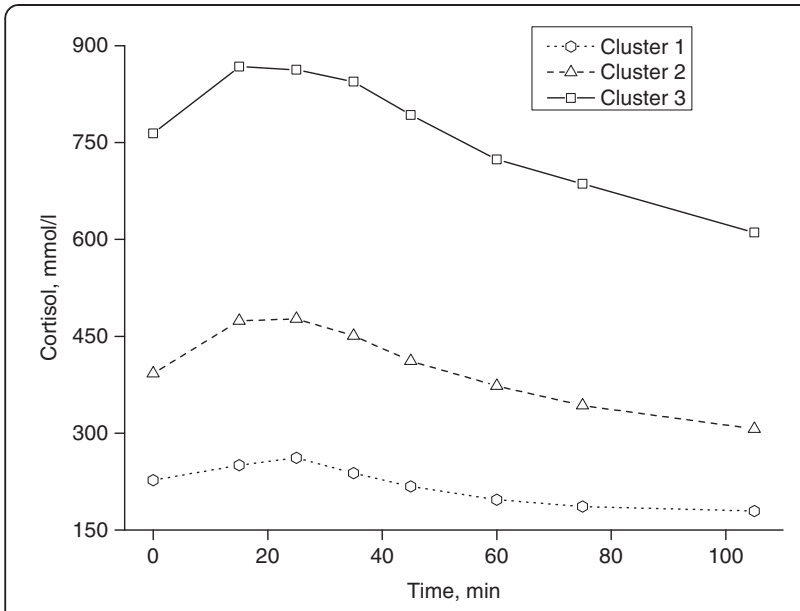

Fig. 1 Actual cortisol values in different cortisol response groups in females

males, displayed a substantial response with a large variation in cortisol values between baseline and peak (178 mmol/L, $183 \mathrm{mmol} / \mathrm{L})$.

Cluster 3 included significantly more females using OC $(87 \%)$ than cluster $2(39 \%)$ or cluster 1 (11 \%) $(p<0.001)$ (Table 2$)$. Males with a high physical activity level were underrepresented in cluster $3(p=0.048)$, and a similar but statistically non-significant trend was seen among females $(p=0.090)$. Obesity $(p=0.073)$ and smoking $(p=0.085)$ tended to be associated with being in cluster 1 among females, but these associations were not statistically significant. Also, there were no significant differences in depressive symptoms across the cortisol response groups.

\section{Association between cortisol response and MS pain}

Compared with cluster 2, belonging to cluster 1 or cluster 3 (i.e., cortisol hypo- or hyper-responsiveness)

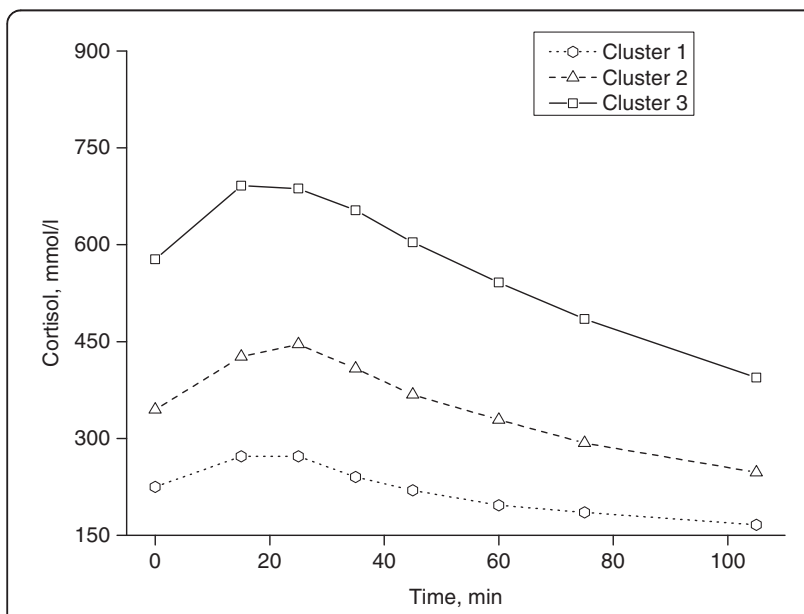

Fig. 2 Actual cortisol values in different cortisol response groups in males was not associated with any MS pain or a high pain problem severity index in either females or males (Tables 3 and 4). The females in cluster 1 demonstrated a significant association with any MS pain (odds ratio 2.3, $95 \%$ confidence interval 1.0-5.0) and with high pain problem severity index $(2.8,1.1-6.8)$ if CPT was above the median. The females in cluster 1 also had an increased likelihood of a high pain problem severity index if PPT was below the median $(3.5,1.3-9.7)$, whereas any MS pain in combination with PPT below the median showed only a borderline significant association (2.2, 1.0-4.8). Among males, all associations remained statistically non-significant (Table 4).

\section{Discussion}

In this study, in females with pain sensitivity measures above the 50th percentile, a hypoactive HPA axis at age 18 years was associated with both the presence and the severity of pain problems at 22 years. This was evidenced by cluster 1 displaying higher odds than cluster 2 for pain presence and pain problem severity in those with higher cold pain sensitivity, as well as higher odds for pain problems in those with higher pressure pain sensitivity. The associations were independent of the effects of OC use, smoking, BMI, physical activity level and depressive symptoms.

Our study is the first analysis of the association between HPA axis stress response and MS pain in young adults. It was conducted in a representative birth cohort and had a longitudinal design with a 4-year follow-up. Cortisol stress response was measured under controlled conditions using a previously established protocol [20]. Cortisol responses to laboratory stress correlated strongly with total cortisol over the day, but not with cortisol awakening response or cortisol slope in a recent study [40]. In this respect, we believe that our cortisol response groups, although based on response during short-term stimuli, reflected the volume of cortisol secretion in repeated real-life stress.

Using GBTM, we identified three groups showing different cortisol stress responses in young males and females. In a recent study, researchers applied the same statistical method with four saliva cortisol samples collected from a group of adolescent females during the TSST [34]. They found normative (59\%), hyporesponsive $(27 \%)$ and hyperresponsive (15\%) groups, which corresponds well with our results. Van Ryzin et al. used GBTM with diurnal cortisol data among preschool children [35] and also found two atypical cortisol patterns (i.e., a hyper-cortisol group [10\%] and a hypo-cortisol group [16 \%]) in addition to a normative group (74\%). In the majority of TSST studies, investigators have used raw cortisol values or calculated area under curve parameters, and have applied the traditional statistical 
Table 2 Descriptive statistics for covariates according to cortisol response groups

\begin{tabular}{|c|c|c|c|c|c|c|c|c|}
\hline & Females, 1 & & & & Males, $n$ & & & \\
\hline & Cluster 1 & Cluster 2 & Cluster 3 & $p$ Value ${ }^{a}$ & Cluster 1 & Cluster 2 & Cluster 3 & $p$ Value ${ }^{a}$ \\
\hline Oral contraceptive use & & & & $<0.001$ & & & & \\
\hline No & $112(89)$ & $106(61)$ & $9(13)$ & & & & & \\
\hline Yes & $14(11)$ & $67(39)$ & $58(87)$ & & & & & \\
\hline Smoking & & & & 0.085 & & & & 0.300 \\
\hline No & $107(85)$ & $153(89)$ & $64(96)$ & & $74(79)$ & $190(81)$ & $91(87)$ & \\
\hline Yes & $19(15)$ & $19(11)$ & $3(5)$ & & $20(21)$ & $45(19)$ & $14(13)$ & \\
\hline Body mass index & & & & 0.073 & & & & 0.955 \\
\hline Normal & $83(67)$ & $128(74)$ & $50(76)$ & & $67(73)$ & $171(73)$ & $75(71)$ & \\
\hline Overweight & $18(15)$ & $28(16)$ & $12(18)$ & & $16(17)$ & $41(18)$ & $22(21)$ & \\
\hline Obese & $23(19)$ & $16(9)$ & $4(6)$ & & $9(10)$ & $23(10)$ & $9(9)$ & \\
\hline Physical activity level & & & & 0.090 & & & & 0.048 \\
\hline $0 \mathrm{~h} / \mathrm{wk}$ & $50(48)$ & $57(40)$ & $32(53)$ & & $26(38)$ & $49(27)$ & $33(38)$ & \\
\hline $0.1-4.9 \mathrm{~h} / \mathrm{wk}$ & $31(30)$ & $57(40)$ & $24(39)$ & & $18(26)$ & $63(34)$ & $33(38)$ & \\
\hline$\geq 5.0 \mathrm{~h} / \mathrm{wk}$ & $23(22)$ & $28(20)$ & $5(8)$ & & $25(36)$ & $72(39)$ & $20(23)$ & \\
\hline Depressive symptoms & & & & 0.393 & & & & 0.167 \\
\hline Minimal & $73(70)$ & $103(73)$ & $43(75)$ & & $68(91)$ & $171(86)$ & $70(82)$ & \\
\hline Mild & $12(11)$ & $22(16)$ & $8(14)$ & & $2(3)$ & $20(10)$ & $11(13)$ & \\
\hline Moderate to severe & $20(19)$ & $16(11)$ & $6(11)$ & & $5(7)$ & $7(4)$ & $4(5)$ & \\
\hline
\end{tabular}

${ }^{a} p$ Values derived from $x^{2}$ test

techniques [11, 12]. These methods assume sample homogeneity with respect to change over time, whereas underlying subgroups representing different patterns of change can be captured with the group-based trajectory approach [37].

Our findings are broadly in line with previous studies linking HPA axis dysfunction with MS pain [8-12] and increased pain sensitivity [5, 13]. To our knowledge, there are only a few previous studies on the relationship between cortisol response in the TSST and MS pain [11, 12]. One study showed decreased salivary cortisol levels after awakening but not during the TSST among adolescents with overtiredness, dizziness and MS pain [12]. However, the association of MS pain and cortisol levels was not analysed separately from other symptoms, and the TSST was preceded by other potentially stressful tasks. In a small-scale study, middle-aged patients with fibromyalgia showed significantly reduced plasma cortisol response during the TSST, supporting our findings [11]. A flattened diurnal cortisol profile predicted newonset widespread pain among individuals with high somatization and health-seeking behaviour in a British study [10], and another population-based study also showed that chronic multi-site MS pain was associated with lower cortisol levels and blunted diurnal slope [8]. The researchers in these two studies did not analyse stress-related cortisol levels, however, and the findings are not directly comparable to ours. In one study, researchers did not detect an association between 24-h urinary free cortisol and functional MS symptoms [41].

Interestingly, we found that a hyporesponsive HPA axis was significantly associated with MS pain in females only in combination with increased pain sensitivity. A population-based sample of young adults with MS pain is likely to be heterogeneous, with some having injuryor disease-related pain and others with more nonspecific symptoms. Relative hypocortisolism may explain pain in certain subgroups by enhancing both central and peripheral sensitization of pain pathways by increased pro-inflammatory activity [42, 43]. Among patients with back pain, increased levels of pro-inflammatory cytokines together with a hyporesponsive HPA axis predicted failure in surgery [9], whereas patients with chronic widespread pain showed lower levels of Th2 anti-inflammatory cytokines than controls [44]. Proinflammatory cytokines may activate' a sickness response', which is believed to explain typical symptoms in functional pain syndromes, including exaggerated pain sensitivity [43]. This potential underlying mechanism supports our results, assuming that pain hypersensitivity is a marker of sensitization of the nervous system.

We did not find any association between HPA axis function and MS pain in males. Previous studies have 
Table 3 Multivariate logistic regression analysis for variables associated with musculoskeletal pain alone and combined with increased pain sensitivity among females

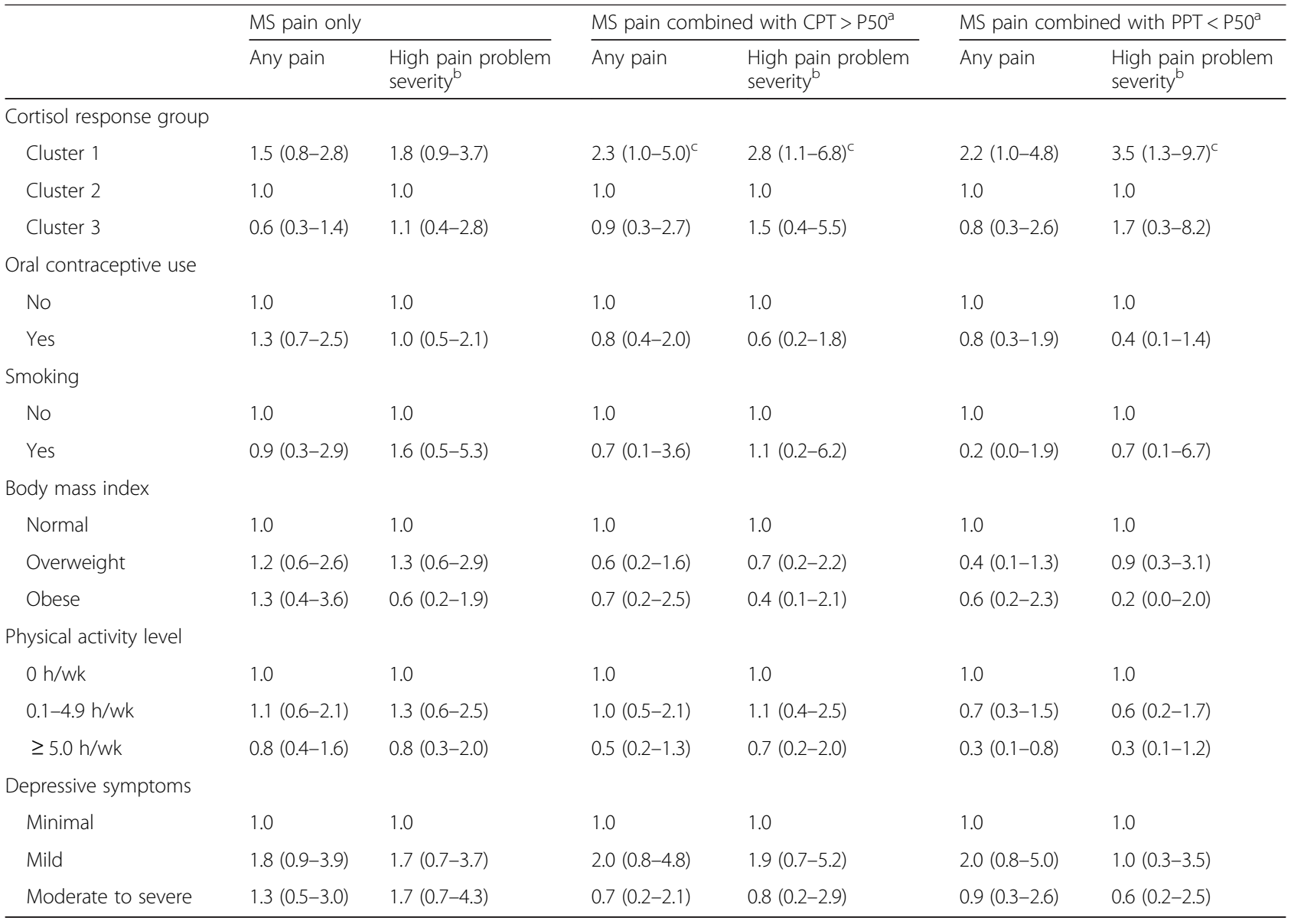

MS musculoskeletal, CPT cold pain threshold, PPT pressure pain threshold

Data are presented as odds ratios with $95 \%$ confidence intervals

${ }^{a}$ Median (P50) CPT $=15^{\circ} \mathrm{C}$; median PPT $=318 \mathrm{kPa}$

${ }^{\mathrm{b}}$ High pain problem severity index $=15$ or more

${ }^{c} p<0.05$

All covariates were included in the multivariate model. Reference group includes those without pain or with a low severity of pain and those with pain but with CPT $<$ P50 or PPT $>$ P50

The number of individuals included in the analysis varied from 198 to 222 due to missing data

reported that, compared with males, females have more chronic pain disorders [1], a lower pain threshold [25] and a lower cortisol response to stress [45]. In our study, cluster 1 had a higher proportion of females (34\%) than males $(21 \%)$, and females showed lower cortisol values during the TSST than males after controlling for OC use (data not shown). This lower cortisol response, as a sign of relative hypocortisolism, observed in females more often than males may contribute to the higher prevalence of pain disorders in females [45], and also explains why we did not find a significant association in males. The sex hormones may have a role in the reported differences between sexes [45]. Oestradiol has been shown to induce HPA axis stimulation [46] and also to improve pain perception [47]. Although females generally show lower HPA axis response to stress than males of the same age [45], the response during the luteal menstrual phase (high oestrogen level) is higher than during the follicular phase (low oestrogen level) and is comparable to that of males [48]. An explanation for our findings of sex differences warrants further studies controlling for menstrual cycle phase.

An important limitation of our study is that we did not have information about menstrual cycle phase, which may potentially confound the results. Variation in oestrogen levels influencing cortisol binding capacity is likely to explain intra- and inter-individual differences among females [28], such as our finding that OC use is associated with a higher total plasma cortisol response in the TSST. We were not able to analyse females on OC separately, owing to sample size restrictions. As excluding females on $\mathrm{OC}$ did not change the overall results 
Table 4 Multivariate logistic regression analysis for variables associated with musculoskeletal pain alone and combined with increased pain sensitivity among males

\begin{tabular}{|c|c|c|c|c|c|c|}
\hline & \multicolumn{2}{|l|}{ MS pain only } & \multicolumn{2}{|c|}{ MS pain combined with $\mathrm{CPT}>{\mathrm{P} 50^{\mathrm{a}}}$} & \multicolumn{2}{|c|}{ MS pain combined with PPT $<$ P50 } \\
\hline & Any pain & $\begin{array}{l}\text { High pain problem } \\
\text { severity }^{\mathrm{b}}\end{array}$ & Any pain & $\begin{array}{l}\text { High pain problem } \\
\text { severity }^{\mathrm{b}}\end{array}$ & Any pain & $\begin{array}{l}\text { High pain problem } \\
\text { severity }^{\mathrm{b}}\end{array}$ \\
\hline \multicolumn{7}{|l|}{ Cortisol response group } \\
\hline Cluster 1 & $0.7(0.3-1.5)$ & $0.6(0.3-1.5)$ & $1.2(0.4-3.3)$ & $1.6(0.5-4.9)$ & $1.5(0.6-4.1)$ & $1.7(0.6-4.9)$ \\
\hline Cluster 2 & 1.0 & 1.0 & 1.0 & 1.0 & 1.0 & 1.0 \\
\hline Cluster 3 & $0.9(0.4-1.8)$ & $0.8(0.3-1.8)$ & $1.0(0.4-2.9)$ & $1.2(0.3-3.9)$ & $2.1(0.9-5.4)$ & $2.1(0.7-5.9)$ \\
\hline \multicolumn{7}{|l|}{ Smoking } \\
\hline No & 1.0 & 1.0 & 1.0 & 1.0 & 1.0 & 1.0 \\
\hline Yes & $1.1(0.4-2.8)$ & $1.7(0.6-4.4)$ & $0.2(0.0-1.9)$ & $0.3(0.0-2.9)$ & $0.6(0.1-2.8)$ & $0.7(0.2-3.7)$ \\
\hline \multicolumn{7}{|l|}{ Body mass index } \\
\hline Normal & 1.0 & 1.0 & 1.0 & 1.0 & 1.0 & 1.0 \\
\hline Overweight & $1.5(0.7-3.0)$ & $1.8(0.8-3.8)$ & $0.9(0.3-2.6)$ & $1.1(0.4-3.6)$ & $0.7(0.3-2.0)$ & $1.0(0.3-3.1)$ \\
\hline Obese & $1.7(0.5-6.2)$ & $2.9(0.8-10.8)$ & $1.1(0.1-9.5)$ & $1.6(0.2-15.8)$ & $1.5(0.3-7.7)$ & $2.2(0.4-11.7)$ \\
\hline \multicolumn{7}{|l|}{ Physical activity level } \\
\hline $0 \mathrm{~h} / \mathrm{wk}$ & 1.0 & 1.0 & 1.0 & 1.0 & 1.0 & 1.0 \\
\hline $0.1-4.9 \mathrm{~h} / \mathrm{wk}$ & $0.7(0.3-1.4)$ & $0.4(0.2-1.0)$ & $0.4(0.1-1.3)$ & $0.3(0.1-1.2)$ & $0.8(0.3-2.1)$ & $0.6(0.2-1.8)$ \\
\hline$\geq 5.0 \mathrm{~h} / \mathrm{wk}$ & $1.0(0.5-2.1)$ & $0.8(0.4-1.8)$ & $0.8(0.3-2.0)$ & $0.8(0.3-2.4)$ & $0.8(0.3-2.1)$ & $0.9(0.3-2.5)$ \\
\hline \multicolumn{7}{|l|}{ Depressive symptoms } \\
\hline Minimal & 1.0 & 1.0 & 1.0 & 1.0 & 1.0 & 1.0 \\
\hline Mild & $1.4(0.5-4.2)$ & $1.6(0.5-4.9)$ & $3.1(0.9-10.8)$ & $3.3(0.9-12.8)$ & $1.4(0.3-5.5)$ & $1.8(0.4-7.5)$ \\
\hline Moderate to severe & $4.2(1.0-17.9)$ & $3.7(0.9-15.3)$ & $1.2(0.1-10.9)$ & $1.5(0.2-15.2)$ & $0.7(0.1-6.4)$ & $0.9(0.1-8.4)$ \\
\hline
\end{tabular}

MS musculoskeletal, CPT cold pain threshold, $P P T$ pressure pain threshold

Data are presented as odds ratios with $95 \%$ confidence intervals

${ }^{\mathrm{a}}$ Median (P50) $\mathrm{CPT}=7{ }^{\circ} \mathrm{C}$; median PPT $=419 \mathrm{kPa}$

${ }^{\mathrm{b}}$ High pain problem severity index $=9$ or more

All covariates were included in the multivariate model. Reference group includes those without pain or with a low severity of pain and those with pain but with CPT $<$ P50 or PPT $>$ P50

The number of individuals included in the analysis varied from 198 to 215 due to missing data

(non-reported data), however, all females were analysed together. As cortisol values were higher at the beginning than at the end of the TSST in all response groups, a start point of HPA axis activation and true baseline values cannot be determined. Although cluster 1 and 3 were interpreted as hyporesponsive and hyperresponsive, we cannot exclude that the groups reflect different baseline levels rather than differential responsiveness. Unfortunately, we did not have subjective or objective measures of stress related to anticipation or cannulation during the rest phase, nor did we have previous data on baseline cortisol levels.

Potential fluctuation of HPA axis function over an extended time remains unclear. The TSST has been performed on only one occasion in the Raine cohort. HPA axis hyporeactivity appeared stable over 12 months among women with depression, with authors of this particular study suggesting that cortisol hyporesponse may be a trait marker [49]. During childhood and adolescence, the long-term stability of HPA axis function in stress has been shown to be at least mild to moderate
$[50,51]$, potentially supporting this view. However, while we consider that HPA axis function at 18 years of age may be related to a pro-nociceptive state that increases the potential for altered pain sensitivity and MS pain later in life, stability of HPA axis function over time may moderate this assumption. Another limitation is that we did not assess pain status or pain thresholds at baseline, leaving their course unknown. Consequently, we could not explicitly determine the causality of the observed associations (i.e., whether HPA axis dysfunction was a cause rather than a biomarker of MS pain). Future research using multiple measures of cortisol and MS pain over time is required to further test the validity of our results.

Our measure of pain problem severity was limited to five questions derived from the well-validated Örebro Musculoskeletal Pain Questionnaire, and we believe it demonstrates face validity as it includes multiple facets of a pain problem. However, we acknowledge that this measure has not previously been used or validated against other more established measures of the severity of pain conditions. We were not able to test cut-off 
values other than the median for pain thresholds, because sample sizes in each subgroup would have become too small. Finally, due to the large number of participants, we were restricted to capture of pain thresholds only, without using dynamic tests of pain sensitivity, which presents a somewhat limited assessment of the pain-processing system [52].

\section{Conclusions}

We identified that a hyporesponsive HPA axis at 18 years of age is associated with MS pain at age 22 years in young adults. However, the association between cortisol stress response and MS pain was found only in females and when pain was related to increased pain sensitivity. In the future, studies with longitudinal cortisol data and MS pain would be useful to further elucidate the nature of the relationship between HPA axis function and MS pain, including the sex discrepancy.

\section{Abbreviations \\ BIC: Bayesian information criterion; BMI: body mass index; CPT: cold pain threshold; GBTM: group-based trajectory modelling; HPA: hypothalamic- pituitary-adrenal; HREC: human research ethics committee; \\ MS: musculoskeletal; OC: oral contraceptive; PPT: pressure pain threshold: SD: standard deviation; TSST: Trier Social Stress Test.}

\section{Competing interests}

The authors declare that they have no competing interests.

\section{Authors' contributions}

$M P, P O, L S, D B, P C, J K, C P$ and $A S$ participated in study conception and design and contributed to the writing of the manuscript. MP was responsible for the literature review. POS, LS, DB, CP and AS were involved in data collection. MP and AS performed statistical analyses. MP drafted the first manuscript. All the authors meet the criteria for authorship, and all read and approved the final manuscript.

\section{Acknowledgements}

The authors thank the Raine Study participants for their ongoing participation in the study; the Raine Study Team for study co-ordination and data collection. The authors also thank the following entities for providing funding for the Core Management of the Raine Study: the UWA Centre for Science for use of the facility; the University of Western Australia (UWA); the Raine Medical Research Foundation; the UWA Faculty of Medicine, Dentistry and Health Sciences; the Telethon Kids Institute; the Women's and Infant's Research Foundation (KEMH); Curtin University; and Edith Cowan University. The 22-year Raine Study follow-up was funded by National Health and Medical Research Council (NHMRC) project grants 1027449, 1044840 and 1021855. Funding was also provided by Safe Work Australia. The TSST was funded by the Canadian Institutes of Health Research (Lye et al., grant MOP-82893), and the 17-year follow-up was supported by an NHMRC program grant (Stanley et al., grant 353514). MP was supported by the Emil Aaltonen Foundation, the Signe and Ane Gyllenberg Foundation and the Finnish Association for the Study of Pain. JK was supported by the Yrjö Jahnsson Foundation. DB and LS were supported by research fellowships from the NHMRC of Australia.

\section{Author details}

${ }^{1}$ Centre for Life Course Epidemiology, and Medical Research Centre Oulu, Oulu University Hospital and University of Oulu, Oulu, Finland. ${ }^{2}$ School of Physiotherapy and Exercise Science, Curtin University, Perth, Australia. ${ }^{3}$ Centre of Expertise for Health and Work Ability and Disability Prevention Centre, Finnish Institute of Occupational Health, Oulu, Finland. ${ }^{4}$ School of Women's and Infants' Health, The University of Western Australia, Perth, Australia.
Received: 17 May 2015 Accepted: 25 November 2015

Published online: 10 December 2015

\section{References}

1. Aaron LA, Buchwald D. Chronic diffuse musculoskeletal pain, fibromyalgia and co-morbid unexplained clinical conditions. Best Pract Res Clin Rheumatol. 2003;17:563-74.

2. McBeth J, Jones K. Epidemiology of chronic musculoskeletal pain. Best Pract Res Clin Rheumatol. 2007;21:403-25.

3. Van Houdenhove B, Luyten P. Central sensitivity syndromes: stress system failure may explain the whole picture. Semin Arthritis Rheum. 2009;39:218-9.

4. Crettaz B, Marziniak M, Willeke P, Young P, Hellhammer D, Stumpf A, et al. Stress-induced allodynia-evidence of increased pain sensitivity in healthy humans and patients with chronic pain after experimentally induced psychosocial stress. PLoS One. 2013:8:e69460.

5. Kuehl LK, Michaux GP, Richter S, Schanchinger H, Anton F. Increased basal mechanical pain sensitivity but decreased perceptual wind-up in a human model of relative hypocortisolism. Pain. 2010;149:539-46.

6. KI H, Nicholl BI, Macfarlane GJ, Thomson W, Davies KA, McBeth J. Genetic variation in the hypothalamic-pituitary-adrenal stress axis influences susceptibility to musculoskeletal pain: results from the EPIFUND study. Ann Rheum Dis. 2010;69:556-60.

7. Miller GE, Chen E, Zhou ES. If it goes up, must it come down? Chronic stress and the hypothalamic-pituitary-adrenocortical axis in humans. Psychol Bull. 2007;133:25-45.

8. Generaal E, Vogelzangs N, Macfarlane GJ, Geenen R, Smit JH, Penninx BW, et al. Reduced hypothalamic-pituitary-adrenal axis activity in chronic multi-site musculoskeletal pain: partly masked by depressive and anxiety disorders. BMC Musculoskelet Disord. 2014;15:227.

9. Geiss A, Rohleder N, Kirschbaum C, Steinbach K, Bauer HW, Anton F. Predicting the failure of disc surgery by a hypofunctional HPA axis: evidence from a prospective study on patients undergoing disc surgery. Pain. 2005:114:104-17.

10. McBeth J, Silman AJ, Gupta A, Chiu YH, Ray D, Morriss R, et al. Moderation of psychosocial risk factors through dysfunction of the hypothalamicpituitary-adrenal stress axis in the onset of chronic widespread musculoskeletal pain: findings of a population-based prospective cohort study. Arthritis Rheum. 2007;56:360-71.

11. Wingenfeld K, Heim C, Schmidt I, Wagner D, Meinlschmidt G, Hellhammer DH. HPA axis reactivity and lymphocyte glucocorticoid sensitivity in fibromyalgia syndrome and chronic pelvic pain. Psychosom Med. 2008; $70: 65-72$

12. Janssens KA, Oldehinkel AJ, Verhulst FC, Hunfeld JA, Ormel J, Rosmalen JG. Symptom-specific associations between low cortisol responses and functional somatic symptoms: the TRAlLS study. Psychoneuroendocrinology. 2012;37:332-40.

13. Godfrey KM, Strachan E, Dansie E, Crofford L, Buchwald D, Goldberg J, et al. Salivary cortisol and cold pain sensitivity in female twins. Ann Behav Med. 2014;:47:180-8

14. Generaal E, Vogelzangs N, Macfarlane GJ, Geenen R, Smit JH, de Geus EJ, et al. Biological stress systems, adverse life events and the onset of chronic multisite musculoskeletal pain: a 6-year cohort study. Ann Rheum Dis. doi: 10.1136/annrheumdis-2014-206741.

15. Badanes LS, Watamura SE, Hankin BL. Hypocortisolism as a potential marker of allostatic load in children: associations with family risk and internalizing disorders. Dev Psychopathol. 2011;23:881-96.

16. Hoftun GB, Romundstad PR, Zwart JA, Rygg M. Prevalence of chronic idiopathic musculoskeletal pain and headache in adolescence: a population based Norwegian study [poster]. Pediatr Rheumatol Online J. 2012;10 Suppl 1:A77.

17. Lupien SJ, McEwen BS, Gunnar MR, Heim C. Effects of stress throughout the lifespan on the brain, behaviour and cognition. Nat Rev Neurosci. 2009;10:434-45.

18. Williams LA, Evans SF, Newnham JP. Prospective cohort study of factors influencing the relative weights of the placenta and the newborn infant. BMJ. 1997:314:1864-8.

19. O'Sullivan PB, Beales DJ, Smith AJ, Straker LM. Low back pain in 17 year olds has substantial impact and represents an important public health disorder: a cross-sectional study. BMC Public Health. 2012;12:100. 
20. Kirschbaum C, Pirke KM, Hellhammer DH. The 'Trier Social Stress Test'-a tool for investigating psychobiological stress responses in a laboratory setting. Neuropsychobiology. 1993;28:76-81

21. Dickerson SS, Kemeny ME. Acute stressors and cortisol responses: a theoretical integration and synthesis of laboratory research. Psychol Bull. 2004;130:355-91

22. Foley P, Kirschbaum C. Human hypothalamus-pituitary-adrenal axis responses to acute psychosocial stress in laboratory settings. Neurosci Biobehav Rev. 2010;35:91-6.

23. Linton SJ, Boersma K. Early identification of patients at risk of developing a persistent back problem: the predictive validity of the Örebro Musculoskeletal Pain Questionnaire. Clin J Pain. 2003:19:80-6.

24. Rolke R, Baron R, Maier C, Tölle TR, Treede RD, Beyer A, et al. Quantitative sensory testing in the German Research Network on Neuropathic Pain (DFNS): standardized protocol and reference values. Pain. 2006;123:231-43.

25. Chesterton LS, Barlas P, Foster NE, Baxter GD, Wright CC. Gender differences in pressure pain threshold in healthy humans. Pain. 2003;101:259-66.

26. Paananen M, Auvinen J, Taimela S, Tammelin T, Kantomaa M, Ebeling H, et al. Psychosocial, mechanical, and metabolic factors in adolescents' musculoskeletal pain in multiple locations: a cross-sectional study. Eur J Pain. 2010;14:395-401.

27. Wijnhoven HA, de Vet HC, Smit HA, Picavet HS. Hormonal and reproductive factors are associated with chronic low back pain and chronic upper extremity pain in women-the MORGEN study. Spine. 2006:31:1496-502.

28. Gaffey AE, Wirth MM, Hoks RM, Jahn AL, Abercrombie HC. Circulating cortisol levels after exogenous cortisol administration are higher in women using hormonal contraceptives: data from two preliminary studies. Stress. 2004;17:314-20.

29. Kudielka BM, Wüst S. Human models in acute and chronic stress: assessing determinants of individual hypothalamus-pituitary-adrenal axis activity and reactivity. Stress. 2010;13:1-14.

30. Rimmele U, Zellweger BC, Marti B, Seiler R, Mohiyeddini C, Ehlert U, et al Trained men show lower cortisol, heart rate and psychological responses to psychosocial stress compared with untrained men. Psychoneuroendocrinology. 2007;32:627-35.

31. Cole TJ, Bellizzi MC, Flegal KM, Dietz WH. Establishing a standard definition for child overweight and obesity worldwide: international survey. BMJ. 2000:320:1240-3

32. Craig CL, Marshall AL, Sjöström M, Bauman AE, Booth ML, Ainsworth BE. International physical activity questionnaire: 12-country reliability and validity. Med Sci Sports Exerc. 2003;35:1381-95.

33. Beck AT, Beamesderfer A. Assessment of depression: the depression inventory. Mod Probl Pharmacopsychiatry. 1974;7:151-69.

34. Nagin DS. Group-based modeling of development. Cambridge, MA: Harvard University Press; 2005

35. Van Ryzin MJ, Chatham M, Kryzer E, Kertes DA, Gunnar MR. Identifying atypical cortisol patterns in young children: the benefits of group-based trajectory modeling. Psychoneuroendocrinology. 2009;34:50-61.

36. Giletta M, Calhoun CD, Hastings PD, Rudolph KD, Nock MK, Prinstein MJ. Multi-level risk factors for suicidal ideation among at-risk adolescent females: the role of hypothalamic-pituitary-adrenal axis responses to stress. Abnorm Child Psychol. 2015;43:807-20.

37. Nagin DS, Odgers CL. Group-based trajectory modeling in clinical research. Annu Rev Clin Psychol. 2010;6:109-38.

38. Goldberg R, Gore JM, Barton B, Gurwitz J. Individual and composite study endpoints: separating the wheat from the chaff. Am J Med. 2014;127:379-84

39. Moulin DE, Clark AJ, Gordon A, Lynch M, Morley-Forster PK, Nathan H, et al Long-term outcome of the management of chronic neuropathic pain: a prospective observational study. J Pain. 2015;16:852-61.

40. Kidd T, Carvalho LA, Steptoe A. The relationship between cortisol responses to laboratory stress and cortisol profiles in daily life. Biol Psychol. 2014;99:34-40.

41. Tak LM, Bakker SJ, Rosmalen JG. Dysfunction of the hypothalamic-pituitaryadrenal axis and functional somatic symptoms: a longitudinal cohort study in the general population. Psychoneuroendocrinology. 2009;34:869-77.

42. Chrousos GP. Stress, chronic inflammation, and emotional and physical well-being: concurrent effects and chronic sequelae. J Allergy Clin Immunol. 2000;106(5 Suppl):S275-91.

43. Wieseler-Frank J, Maier SF, Watkins LR. Central proinflammatory cytokines and pain enhancement. Neurosignals. 2005;14:166-74.
44. Uçeyler N, Valenza R, Stock M, Schedel R, Sprotte G, Sommer C. Reduced levels of antiinflammatory cytokines in patients with chronic widespread pain. Arthritis Rheum. 2006;54:2656-64.

45. Kudielka BM, Kirschbaum C. Sex differences in HPA axis responses to stress: a review. Biol Psychol. 2005;69:113-32.

46. Kirschbaum C, Schommer N, Federenko I, Gaab J, Neumann O, Oellers M, et al. Short-term estradiol treatment enhances pituitary adrenal axis and sympathetic responses to psychosocial stress in healthy young men. J Clin Endocrinol Metab. 1996:81:3639-43.

47. Rezaii T, Hirschberg AL, Carlström K, Ernberg M. The influence of menstrual cycle phases on pain modulation in healthy women. J Pain. 2012;13:646-55.

48. Kirschbaum C, Kudielka BM, Gaab J, Schommer NC, Hellhammer DH. Impact of gender, menstrual cycle phase, and oral contraceptives on the activity of the hypothalamus-pituitary-adrenal axis. Psychosom Med. 1999;61:154-62.

49. Wahlberg K, Ghatan PH, Modell S, Nygren A, Ingvar M, Asberg M, et al. Suppressed neuroendocrine stress response in depressed women on job-stress-related long-term sick leave: a stable marker potentially suggestive of preexisting vulnerability. Biol Psychiatry. 2009;65:742-7.

50. Hankin BL, Badanes LS, Smolen A, Young JF. Cortisol reactivity to stress among youth: stability over time and genetic variants for stress sensitivity. J Abnorm Psychol. 2015:124:54-67.

51. Platje E, Vermeiren RR, Branje SJ, Doreleijers TA, Meeus WH, Koot HM, et al. Long-term stability of the cortisol awakening response over adolescence. Psychoneuroendocrinology. 2013;38:271-80.

52. Arendt-Nielsen L, Yarnitsky D. Experimental and clinical applications of quantitative sensory testing applied to skin, muscles and viscera. J Pain. 2009;10:556-72.

\section{Submit your next manuscript to BioMed Central and we will help you at every step:}

- We accept pre-submission inquiries

- Our selector tool helps you to find the most relevant journal

- We provide round the clock customer support

- Convenient online submission

- Thorough peer review

- Inclusion in PubMed and all major indexing services

- Maximum visibility for your research

Submit your manuscript at www biomedcentral com/submit

C Biomed Central 